\title{
Intramuscular schwannoma: clinical and magnetic resonance imaging features
}

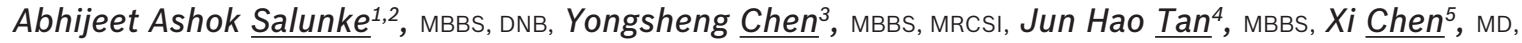 \\ Tun-Lin Foo ${ }^{5}$, MBBS, MRCS, Louise Elizabeth Gartner ${ }^{6}$, MBBS, FRCR, Mark Edward Puhaindran ${ }^{1}$, MBBS, MRCS
}

INTRODUCTION Schwannomas that arise within the muscle plane are called intramuscular schwannomas. The low incidence of these tumours and the lack of specific clinical features make preoperative diagnosis difficult. Herein, we report our experience with intramuscular schwannomas. We present details of the clinical presentation, radiological diagnosis and management of these tumours.

METHODS Between January 2011 and December 2013, 29 patients were diagnosed and treated for histologically proven schwannoma at the National University Hospital, Singapore. Among these 29 patients, eight (five male, three female) had intramuscular schwannomas.

RESULTS The mean age of the eight patients was 40 (range 27-57) years. The most common presenting feature was a palpable mass. The mean interval between surgical treatment and the onset of clinical symptoms was 17.1 (range 4-72) months. Six of the eight tumours $(75.0 \%)$ were located in the lower limb, while $2(25.0 \%)$ were located in the upper limb. None of the patients had any preoperative neurological deficits. Tinel's sign was present in one patient. Magnetic resonance (MR) imaging showed that the findings of split-fat sign, low signal margin and fascicular sign were present in all patients. The entry and exit sign was observed in $4(50.0 \%)$ patients, a hyperintense rim was observed in 7 (87.5\%) patients and the target sign was observed in $5(62.5 \%)$ patients. All patients underwent microsurgical excision of the tumour and none developed any postoperative neurological deficits.

CONCLUSION Intramuscular schwannomas demonstrate the findings of split-fat sign, low signal margin and fascicular sign on MR imaging. These findings are useful for the radiological diagnosis of intramuscular schwannoma.

Keywords: intramuscular, magnetic resonance imaging, schwannoma

\section{INTRODUCTION}

Schwannomas are benign neurogenic tumours that arise from nerve sheath cells. They present in the second to fifth decade of life, with a prevalence of $5 \%$ among all benign soft tissue neoplasms. ${ }^{(1-4)}$ Intramuscular schwannomas are rare tumours. Patients with intramuscular schwannomas present with a slowgrowing soft tissue mass, and may or may not have associated neurological symptoms. The lack of specific clinical and radiological features renders the preoperative diagnosis of intramuscular schwannoma especially challenging. Herein, we report our experience with intramuscular schwannomas. We present details on the clinical presentation, diagnosis and management of these tumours, with an emphasis on a number of radiological features we have observed that may aid in the preoperative diagnosis of intramuscular schwannoma.

\section{METHODS}

Between January 2011 and December 2013, a total of 29 patients were diagnosed and treated for schwannoma at the National University Hospital, Singapore. Of these 29 patients, eight patients (five male, three female) had intramuscular schwannomas. These eight patients had a mean age of 40 (range 27-57) years. The clinical records, magnetic resonance (MR) images, operation notes and histology report of the eight patients were retrospectively reviewed. In all eight patients, the diagnosis of intramuscular schwannoma was confirmed on histology. The following clinical features were reviewed: presence of swelling or mass, neurological symptoms, interval between onset of symptoms and surgery, and presence of Tinel's sign. A consultant musculoskeletal radiologist assessed the MR imaging signs to determine the tumour size, as well as the presence or absence of the split-fat sign, target sign, fascicular sign, entry and exit sign, low signal margin and hyperintense rim.

\section{RESULTS}

The demographic and clinical features of the eight patients with intramuscular schwannomas are shown in Table I. All eight patients presented with a palpable mass or swelling. A mean interval of 17.1 (range 4-72) months was noted between the onset of clinical symptoms and surgical treatment. In six patients, the intramuscular schwannoma was found in the lower limb, while in two patients, it was found in the upper limb. None of the patients had preoperative neurological deficits, although Tinel's sign was elicited in one patient.

The results of MR imaging by the consultant musculoskeletal radiologist showed that the split-fat sign, a low signal margin and the fascicular sign were present in all the patients who had intramuscular schwannomas (Table II). The entry and exit 
Table I. Demographics and clinical features of the patients with intramuscular schwannomas $(n=8)$.

\begin{tabular}{lclclcc}
\hline Patient no. & Age $(\mathbf{y r})$ & Gender & Duration of symptoms (mth) & Palpable mass & Tinel's sign & Follow-up duration (mth) \\
\hline 1 & 27 & Female & 4 & Yes & Absent \\
2 & 27 & Female & 42 & Yes & Absent \\
3 & 57 & Male & 5 & Yes & Absent \\
4 & 36 & Male & 72 & Yes & Absent \\
5 & 60 & Female & 12 & Yes & Absent \\
6 & 36 & Male & 24 & Yes & Absent \\
7 & 35 & Male & 12 & Yes & Present \\
8 & 47 & Male & 4 & Yes & 9 \\
\hline
\end{tabular}

Table II. Magnetic resonance imaging findings of the patients with intramuscular schwannomas $(n=8)$.

\begin{tabular}{|c|c|c|c|c|c|c|}
\hline Patient no. & Split-fat sign & Fascicular sign & Low signal margin & Ring enhancement & Target sign & Muscle involved \\
\hline 1 & Present & Present & Present & $\mathrm{Nil}$ & Nil & Soleus \\
\hline 2 & Present & Present & Present & Present & Present & Dorsal interosseous \\
\hline 4 & Present & Present & Present & Present & Present & Extensor carpi ulnaris \\
\hline 5 & Present & Present & Present & Present & Present & Gracilis \\
\hline 6 & Present & Present & Present & Present & Nil & Gastrocnemius \\
\hline 7 & Present & Present & Present & Present & Present & Peroneus longus \\
\hline 8 & Present & Present & Present & Present & Present & Soleus \\
\hline
\end{tabular}

sign was observed in $4(50.0 \%)$ patients and a hyperintense rim was observed in 7 (87.5\%) patients (Figs. 1 \& 2). The target sign was noted in $5(62.5 \%)$ patients (Fig. 3). All eight patients underwent microsurgical excision and histological confirmation of the tumour. None of the patients developed any postoperative neurological deficits.

\section{DISCUSSION}

Schwannomas can be classified by their anatomical location. Specific subtypes include intermuscular schwannomas, intramuscular schwannomas, subcutaneous schwannomas and intraosseous schwannomas. ${ }^{(4,5)}$ Intramuscular schwannomas are situated within the muscle tissues, while intermuscular schwannomas are situated between the muscle tissues.

Intramuscular schwannomas typically present as a slow-growing mass or a swelling in the extremities. ${ }^{(4-8)}$ There is usually a long interval between the onset of clinical symptoms and treatment, as these lesions are slow-growing and usually situated deep within the muscles. Patients rarely present with neurological symptoms (i.e. tingling, numbness and motor or sensory loss), ${ }_{1}^{(4,5)}$ and the mass is usually non-tender, firm in consistency and nontransilluminant. Tinel's sign is not classically elicited in patients with intramuscular schwannomas, as the tumour, which is deeply located, usually arises from small motor nerve branches within the substance of muscles. ${ }^{(4-8)}$ Specific motor weakness due to motor branch involvement is also uncommon.

MR imaging has proven useful in the preoperative diagnosis of intramuscular schwannoma. ${ }^{(3-5)}$ In the present study, we found that the most helpful MR imaging findings that supported the diagnosis of intramuscular schwannoma were the split-fat sign, a low signal margin, the fascicular sign and a hyperintense rim. The first three MR imaging features were identified in all the patients

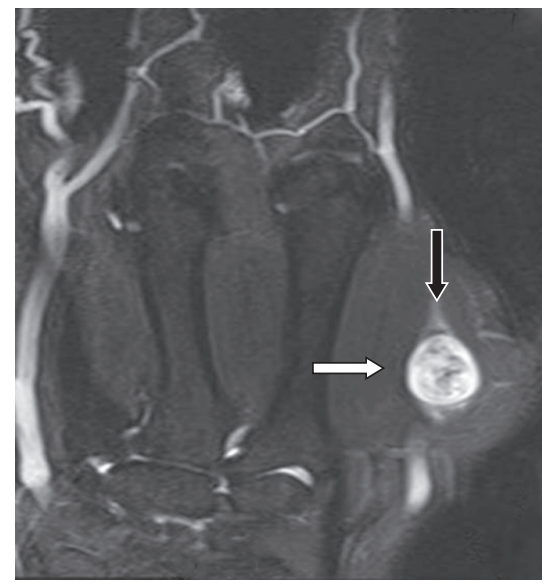

Fig. 1 Coronal T2-W fat-suppressed MR image shows an intramuscular schwannoma in the first dorsal interosseous muscle of the hand. The split-fat sign (white arrow) and the entry and exit sign (black arrow) are visible.

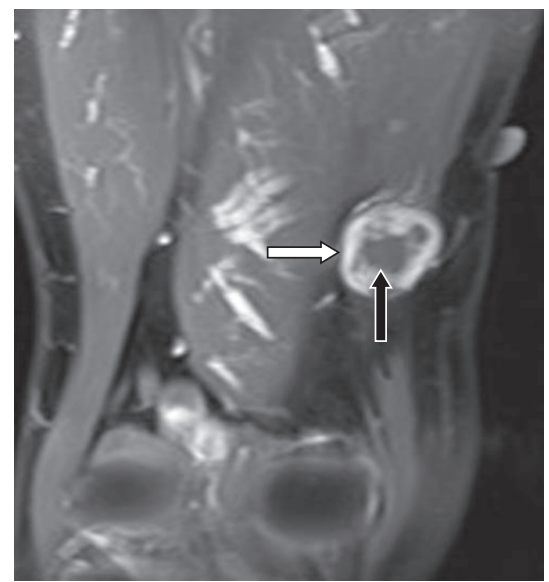

Fig. 2 Coronal T1-W fat-suppressed MR image shows ring enhancement, a peripheral hyperintense rim (white arrow) and central hypointense signal (black arrow). 


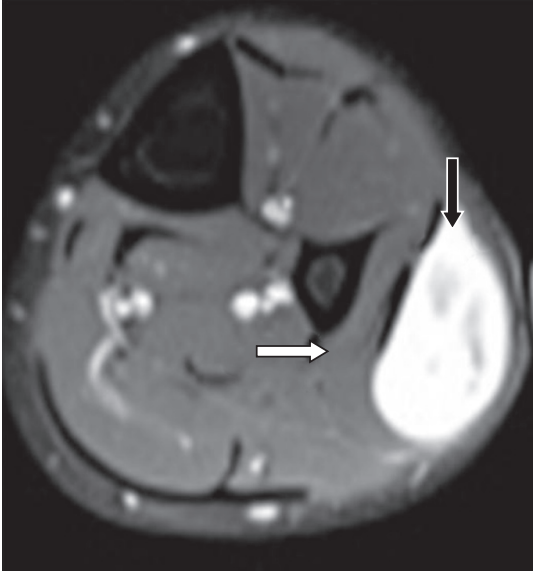

Fig. 3 Axial T1-W fat-suppressed MR image shows an intramuscular schwannoma in the peroneus longus muscle (white arrow), with target sign (black arrow).

in the present study, while a hyperintense rim was observed in $87.5 \%$ of the patients.

The split-fat sign is identified by the detection of prominent fat tissue surrounding a nerve tumour; it is usually better appreciated on T1-weighted images. (3,4,-11) $^{(2)}$ is feature develops because the neurovascular bundle is normally surrounded by fat. A slow-growing mass arising from the neurovascular bundle therefore tends to maintain a rim of fat around itself. ${ }^{(3)}$ The split-fat sign is a common feature in most benign peripheral nerve sheath tumours. ${ }^{(3-5,7-14)}$ A low signal margin is seen as a low signal rim surrounding the schwannoma on T2-weighted images; it corresponds to the epineurium covering the schwannoma. ${ }^{(3-5,8,10,11,13-17)}$ On the other hand, the fascicular sign is characterised by multiple ring-like structures within the lesion and higher signal intensities in the periphery. It is more easily observed on T2-weighted axial and gadolinium-enhanced MR images. ${ }^{(3,10,11,15)} \mathrm{A}$ hyperintense rim refers to a thin peripheral rim enhancement that is seen on T2-weighted MR images; it is due to the greater fluid content in the peripheral myxoid tissue. . $^{(4,5,15-19)}$

The target sign is seen on T2-weighted images as a peripheral hyperintense rim with a central low signal intensity. ${ }^{(4,5,15-19)}$ The central low signal intensity reflects fibrous tissues with Antoni A cells in the centre. Peripherally, myxoid tissues with Antoni $B$ cells that bear a higher fluid content contribute to the high signal intensities seen on T2-weighted images. ${ }^{(4,5,15-19)}$ The entry and exit sign of the nerve is a consistent and commonly seen MR imaging feature of schwannomas. ${ }^{(3,4)}$ A hyperintense signal situated longitudinal to a fusiform mass leads to the formation of the entry and exit sign on T2-weighted MR images. ${ }^{(3,4,10,11,14)}$ The target sign was identified in $62.5 \%$ of the patients in the present study, while the entry and exit sign was identified in $50.0 \%$ of the patients.

MR imaging is helpful in differentiating between schwannomas and neurofibromas. Schwannomas are usually encapsulated and situated in an eccentric position in the nerve, while neurofibromas are not. ${ }^{(17)} \mathrm{MR}$ imaging is also the imaging modality of choice for differentiating malignant peripheral nerve sheath tumours (MPNSTs) from benign nerve tumours. The MR imaging features of fascicular sign, target sign and split-fat sign are absent in
MPNSTs. ${ }^{(3,9,10,14)}$ Features that are suggestive of MPNST include large tumour size, invasion of fat planes, heterogeneity, ill-defined margins and oedema surrounding the lesion. ${ }^{(9,10,14)}$

In the present study, all eight patients with intramuscular schwannomas were treated with microsurgical excision of the tumour. This approach led to excellent preservation of nerve function. We believe that the tumours, which are eccentrically located along the nerve sheath, mostly displaced the nerve fibres without infiltrating them. This renders resection possible without significant compromise to the continuity of the nerve fibres. Complications that have been reported after excision of the schwannoma include worsening pain and neurological symptoms. ${ }^{(4,5)}$ However, none of these complications were observed among our patients.

To conclude, intramuscular schwannomas show the split-fat sign, a low signal margin and the fascicular sign on MR imaging. These findings can be helpful in the radiological diagnosis of intramuscular schwannoma.

\section{REFERENCES}

1. Kransdorf MJ. Benign soft-tissue tumors in a large referral population: distribution of specific diagnoses by age, sex, and location. AJR Am J Roentgenol 1995; 164:395-402.

2. Enzinger FM, Weiss SW. Benign tumors of peripheral nerves. In: Soft tissue tumors, eds. St Louis, MO: Mosby, 1995: 821-88.

3. Murphey MD, Smith WS, Smith SE, Kransdorf MJ, Temple HT. From the archives of the AFIP. Imaging of musculoskeletal neurogenic tumors: radiologic-pathologic correlation. Radiographics 1999; 19:1253-80.

4. Shimose S, Sugita T, Kubo T, et al. Major-nerve schwannomas versus intramuscular schwannomas. Acta Radiol 2007; 48:672-7.

5. Kwon BC, Baek GH, Chung MS, et al. Intramuscular neurilemoma. J Bone Joint Surg Br 2003; 85:723-5.

6. Muramatsu K, Ihara K, Yoshida Y, Taguchi T. Intramuscular schwannoma arising from the psoas major muscle. Clin Neurol Neurosurg 2008; 110:532-3.

7. Hsu YC, Shih YY, Gao HW, Huang GS. Intramuscular schwannoma arising from the psoas muscle presenting with femoral nerve neuropathy. South Med J 2010; 103:477-9.

8. Nishio J, Ueki T, Naito M. Intramuscular schwannoma of the musculocutaneous nerve: An uncommon clinical presentation. Exp Ther Med 2013; 6:164-166.

9. Li CS, Huang GS, Wu HD, et al. Differentiation of soft tissue benign and malignant peripheral nerve sheath tumors with magnetic resonance imaging. Clin Imaging 2008; 32:121-7.

10. Friedrich RE, Kluwe L, Fünsterer C, Mautner VF. Malignant peripheral nerve sheath tumors (MPNST) in neurofibromatosis type 1 (NF1): diagnostic findings on magnetic resonance images and mutation analysis of the NF1 gene. Anticancer Res 2005; 25:1699-702.

11. Gubbay AD, Moschilla G, Gray BN, Thompson I. Retroperitoneal schwannoma: a case series and review. Aust N ZJ Surg 1995; 65:197-200.

12. Claes H, Oyen R, Stessens R, Vereecken R. Solitary benign schwannoma in the psoas muscle. J Urol 1987; 137:753-6.

13. D'Silva KJ, Dwivedi AJ, Barnwell JM. Schwannoma of the psoas major muscle presenting with abdominal and back pain. Dig Dis Sci 2003; 48:1619-21.

14. Bhargava R, Parham DM, Lasater OE, et al. MR imaging differentiation of benign and malignant peripheral nerve sheath tumors: use of the target sign. Pediatr Radiol 1997; 27:124-9.

15. Reynolds DL Jr, Jacobson JA, Inampudi P, et al. Sonographic characteristics of peripheral nerve sheath tumors. AJR Am J Roentgenol 2004; 182:741-4.

16. Verstraete KL, Achten E, De Schepper A, et al. Nerve sheath tumors: evaluation with CT and MR imaging. J Belge Radiol 1992; 75:311-20.

17. Cerofolini E, Landi A, DeSantis G, et al. MR of benign peripheral nerve sheath tumors. J Comput Assist Tomogr 1991; 15:593-7.

18. Suh JS, Abenoza P, Galloway HR, Everson LI, Griffiths HJ. Peripheral (extracranial) nerve tumors: correlation of MR imaging and histologic findings. Radiology 1992; 183:341- 6.

19. Pilavaki M, Chourmouzi D, Kiziridou A, et al. Imaging of peripheral nerve sheath tumors with pathologic correlation: pictorial review. Eur J Radiol 2004; 52:229-39. 